\title{
Contemporary Role of Computational Analysis in Endovascular Treatment for Thoracic Aortic Disease
}

\author{
Guido H.W. van Bogerijen, MD ${ }^{1}$, Jip L. Tolenaar, MD ${ }^{1}$, Michele Conti, PhD², \\ Ferdinando Auricchio, $\mathrm{PhD}^{2}$, Francesco Secchi, MD ${ }^{3}$, Francesco Sardanelli, MD, \\ Frans L. Moll, MD, PhD ${ }^{4}$, Joost A. van Herwaarden, MD, PhD ${ }^{4}$, Vincenzo Rampoldi, MD ${ }^{1}$, \\ Santi Trimarchi, MD, $\mathrm{PhD}^{1 *}$
}

${ }^{1}$ Thoracic Aortic Research Center, Policlinico San Donato IRCCS, University of Milan, Milan, Italy; ${ }^{2}$ Department of Civil Engineering and Architecture, Structural Mechanics Division, University of Pavia, Pavia, Italy; ${ }^{3}$ Department of Radiology, Policlinico San Donato IRCCS, University of Milan, Milan, Italy; and ${ }^{4}$ Department of Vascular Surgery, University Medical Center Utrecht, Utrecht, The Netherlands

\begin{abstract}
In the past decade, thoracic endovascular aortic repair (TEVAR) has become the primary treatment option in descending aneurysm and dissection. The clinical outcome of this minimally invasive technique is strictly related to an appropriate patient/stent graft selection, hemodynamic interactions, and operator skills. In this context, a quantitative assessment of the biomechanical stress induced in the aortic wall due to the stent graft may support the planning of the procedure. Different techniques of medical imaging, like computed tomography or magnetic resonance imaging, can be used to evaluate dynamics in the thoracic aorta. Such information can also be combined with dedicated patient-specific computer-based simulations, to provide a further insight into the biomechanical aspects. In clinical practice, computational analysis might show the development of aortic disease, such as the aortic wall segments which experience higher stress in places where rupture and dissection may occur. In aortic dissections, the intimal tear is usually located at the level of the sino-tubular junction and/or at the origin of the left subclavian artery. Besides, computational models may potentially be used preoperatively to predict stent graft behavior, virtually testing the optimal stent graft sizing, deployment, and conformability, in order to provide the best endovascular
\end{abstract}

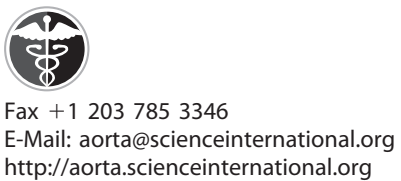

\section{(c) 2013 Aorta.}

Published by Science International Corp. ISSN 2325-4637

Accessible online at:

http://aorta.scienceinternational.org treatment. The present study reviews the current literature regarding the use of computational tools for TEVAR biomechanics, highlighting their potential clinical applications.

Copyright $\odot 2013$ Science International Corp.

\section{Key Words}

Thoracic endovascular aortic repair (TEVAR) - thoracic aortic disease - Computational analysis

\section{Introduction}

In recent years, thoracic endovascular aortic repair (TEVAR) has become a widespread treatment option for thoracic aortic diseases. Compared to open surgery, endovascular repair offers a less invasive approach and its increased availability and applicability have changed the treatment algorithm [1,2]. More than in open surgical repair, the outcome of the endovascular procedure relies on the biomechanical properties of the aortic wall and stent graft. During the cardiac cycle, the force-expressed in Newton (N) by the International System of Units (SI)-acting on the aortic wall is generated by the pulsatility of the

\footnotetext{
*Corresponding author:

Santi Trimarchi, MD, PhD

Thoracic Aortic Research Center

Policlinico San Donato IRCCS

University of Milano

Via Morandi, 30

20097 San Donato Milanese MI, Italy
}

Tel: +3902 52774344, Fax: +3902 52774383, E-Mail: santi.trimarchi@unimi.it 
A
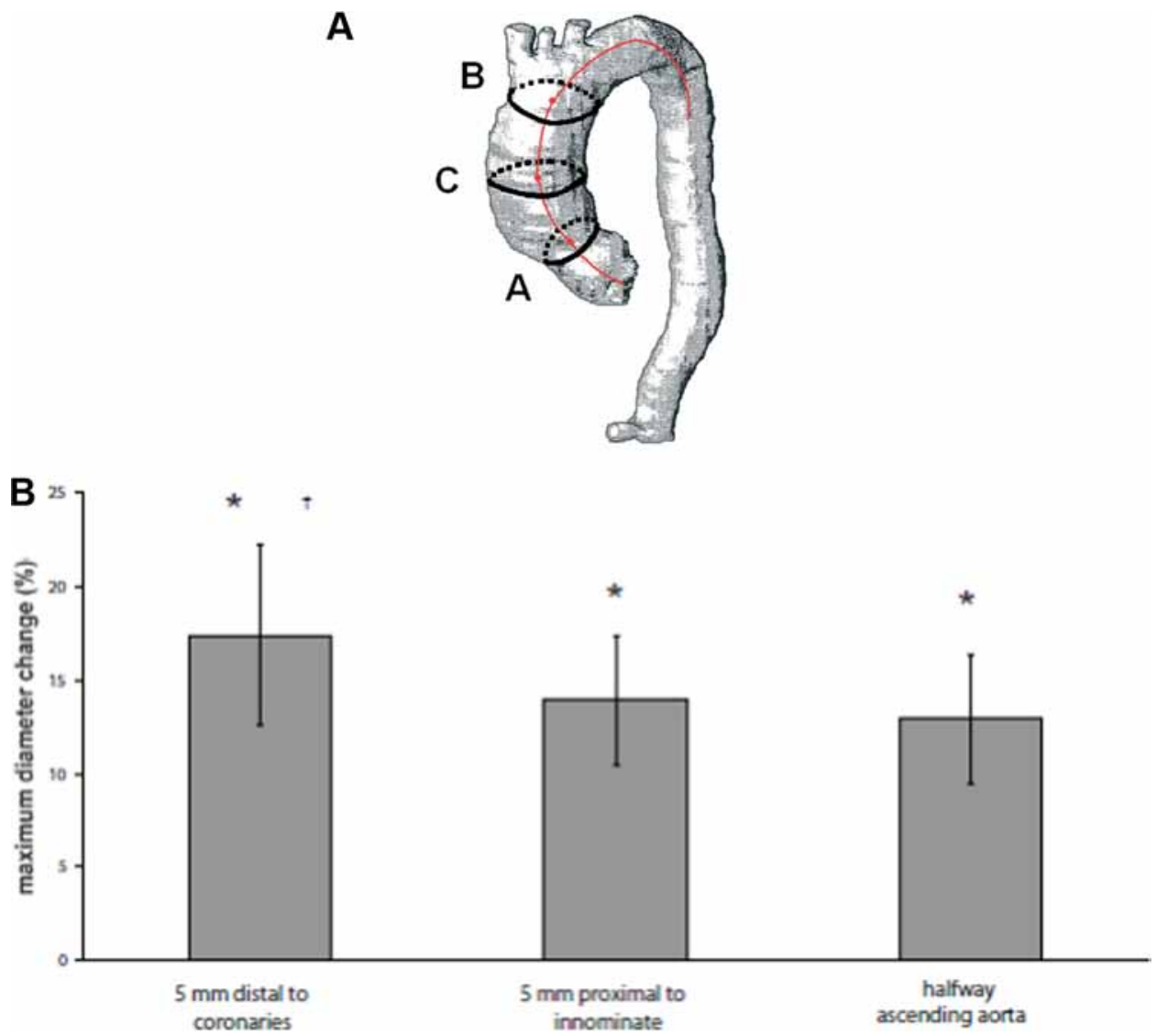

Figure 1. A. The three measured aortic levels with a central lumen line. Level A, $5 \mathrm{~mm}$ distal to the coronary arteries; $B, 5 \mathrm{~mm}$ proximal to the innominate artery; $C$, halfway up the ascending aorta. Figure reproduced with permission of the Journal of Endovascular Therapy (C 2007), provided by Copyright Clearance Center. International Society of Endovascular Specialists. Adapted from van Prehn et al [5]. B. The mean percentage of the maximum diameter change is shown at the 3 different levels. The maximum diameter change at all levels is significant, where level A also differs significantly from levels B and C. Figure reproduced with permission of the Journal of Endovascular Therapy (๔ 2007), provided by Copyright Clearance Center. International Society of Endovascular Specialists. Adapted from van Prehn et al [5].

aorta and its side branches, and by the heartbeat dependent on the plane of movement. The pulsatility in the thoracic aorta varies between the different segments, being higher in the ascending aorta compared to the descending or abdominal aorta, as well as between individual patients [3-5]. The wide range of change in aortic diameter during the cardiac cycle may increase the risk for complications after stent graft placement, such as endoleak or graft migration (Fig. 1A and 1B) $[4,6]$. After deployment of the stent graft, aortic distension is preserved during the cardiac cycle, which may play an important role because repeated stress- expressed in Pascal $\left(\mathrm{Pa}=\mathrm{N}\right.$ per $\left.\mathrm{m}^{2}\right)$ by the $\mathrm{SI}$ - on the stent graft can lead to stent graft fracture or collapse [7]. To prevent these complications, it is important to better understand the forces that contribute to the failure of TEVAR: radial, displacement, and fixation forces. Radial force is achieved by relatively oversizing the stent graft compared to the aortic diameter. A larger radial force will be obtained when larger oversizing is used, while excessive oversizing can lead to stent graft collapse [8]. Displacement force (or drag force) can be defined as the net force due to the action of different stresses that tends to induce stent graft migration. This force is the amount of force required to displace the stent graft, which is determined by stent graft geometry, aortic anatomy, and the hemodynamic condition of the patient [9]. The fixation force can be defined as the resultant or reaction force that counters the so-called displacement force. The combination of such forces will balance the stent graft fixation to the aortic wall, 

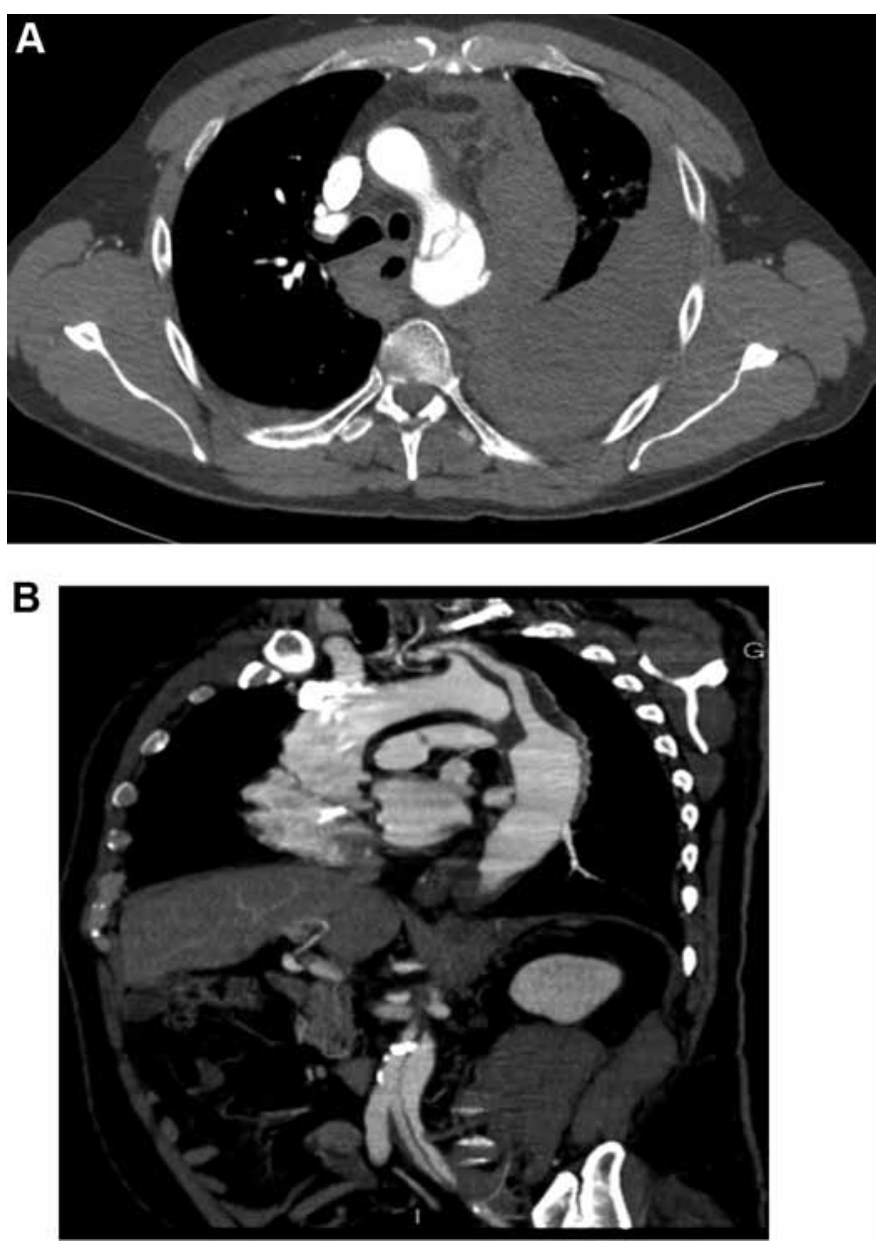

Figure 2. A. CT scan showing an aortic dissection and aortic rupture. B. CT scan showing an aortic dissection with involvement of the left subclavian artery and an aortic aneurysm.

which depends on the aortic morphology (eg, length of proximal and distal landing zone and the condition of the vessel wall) and the mechanical properties of the stent graft (eg, the radial force, presence of barbs and hooks, and the structural rigidity) [10]. Dissection tears or rupture may develop due to a location of high stress in the aortic wall, which can be a result of different parameters, such as the presence of curvature, decreased wall thickness, or resistance and increase in radius, longitudinal stretch, and/or blood pressure. Aortic curvature is physiologically located at the level of the left hemi-arch, which represents a challenging aortic segment to treat with TEVAR. At this level, the presence of concomitant pathological conditions, such as plaques, thrombus, dissections, or aneurysms may further complicate a safe endovascular treatment (Fig. 2A and 2B).
Routinely available imaging techniques, such as computed tomography (CT) and magnetic resonance imaging (MRI), provide detailed information about the aortic morphology, such as angulation, diameter, and length of sealing and fixation zones, which are all characteristics that highly influence the stent graft selection and sizing. However, the forces working on the aortic wall and stent graft cannot be measured with these imaging modalities. Computational modeling has been developed to assess aortic hemodynamic analysis to calculate these forces, which should be taken into account when choosing or designing aortic stent grafts. In this review we report the up-to-date knowledge regarding the contemporary role of computational modeling in thoracic aortic disease, especially for TEVAR, describing the advances in medical imaging, the different components of computational analysis, integration in imaging techniques, and future perspectives.

\section{Advances in Medical Imaging}

Vascular medical imaging has recently evolved with the development of new imaging modalities, which consider the influence of aortic hemodynamics.

Electrocardiographic (ECG) gated CT allows study of the pulsatility of the aorta and stent graft during the cardiac cycle. To obtain images of this kind, it is mandatory to use a scan with an ECG synchronization from 16 to 256 slices. More detectors (slices) result in a reduction of the temporal resolution and, at the same time, a reduction of radiation exposure and contrast materials administration [11,12]. Moreover, iterative reconstruction (IR) algorithms for CT were introduced by all major vendors. These algorithms are noisereducing methods, aimed at either improving image quality using constant $\mathrm{CT}$ radiation dose or lowering the radiation dose without impairing image quality [13]. Dual-energy CT (DECT) imaging discriminates materials based on their varying interactions with photons of different X-ray energies. In the near future, this technique could be further applied to clinical practice for evaluation of the aorta [14]. Actually, a robust computational model can be built using 256 multi-detector CT with a spatial resolution $\leq 1 \mathrm{~mm}$ and a minimum temporal resolution of 8 phases per cardiac cycle.

ECG gated MRI is a technique commonly used in cardiac examination and could be used to evaluate the aortic motion with steady-state free precession 


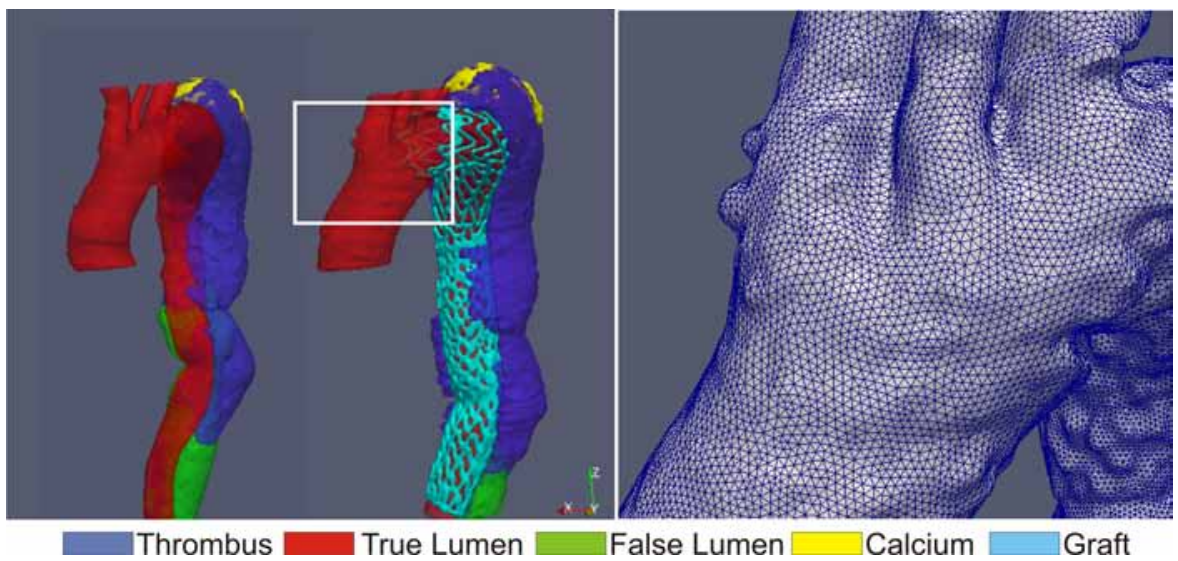

Figure 3. Aortic morphology reconstruction before and after TEVAR as a treatment for chronic type B dissection (left panel). Native mesh (right panel).

sequences, also called bright-blood cine, in several spatial planes. A second dynamic application of MRI is the flow study with phase contrast sequence to evaluate with thought-plane sequences the peak velocity, the forward and reverse flow, and to visualize flow with in-plane sequences. Today, to study aortic diseases, the standard of care is a 1.5-Tesla (T) scan, and the new scanners will probably increase the quality of images obtained by MRI [15]. Several new applications will be ready in the next few years with new sequences and new scans. An example of a new sequence is four-dimensional phase contrast MRI (4D PC-MRI), an emerging tool for the comprehensive evaluation of cardiovascular hemodynamics with full volumetric coverage [16]. The requisite for building an advanced computational model should minimally include a 1.5- to 3-T MRI with a gradient amplitude of 80 $\mathrm{mT} / \mathrm{m}$ and a slew rate gradient of $200 \mathrm{mT} / \mathrm{m} / \mathrm{ms}$.

\section{Computational Analysis}

The introduction of new technologies for treating aortic diseases with endovascular management has resulted in a number of specific issues, ranging from the preoperative phase (ie, a proper patient and stent graft selection), to the postoperative phase (ie, quantitative evaluation of the stent graft performance). Moreover, the significant deviation of pathological aortic anatomy from the healthy (standard) condition calls for patient tailoring of both stent graft design and procedural approach. A successful approach to this complex scenario should consider a real integra- tion of knowledge from different scientific fields, ie, mechanical engineering, informatics, medicine, and more. In this context, computational biomechanics can play a significant role as support to the preoperative planning, through its dedicated computer-based simulations. These might be represented by the approximate resolution of a set of differential equations (eg, incompressible Navier-Stokes), modeling physical phenomena (eg, blood fluid dynamics), in a specific discretized domain, called mesh (eg, a vessel-like geometry). In addition, this approach is aimed at assessing the variation in space and time of primary physiological variables (eg, blood pressure and velocity). Thanks to the impressive technological developments in medical imaging and parallel computing, it is possible to perform complex simulations, embedding patient-specific modeling, potentially compatible with the clinical decision-making process [17]. Such a method consists of three main steps of simulation: 1) preprocessing, 2) solution, and 3) postprocessing.

\section{Preprocessing: From Medical Images to Patient-Specific} Vascular Model

The first step of the simulation set-up involves the creation of mesh, resembling the real physical domain (Fig. 3) [18]. In the specific case of the thoracic aorta, the mesh generation process is not trivial because of its geometrical complexity (aortic arch curvature, branch splitting, etc.). Many dedicated tools (ITKSNAP, 3D Slicer, Osirix, VMTK) are currently available to semi-automatically segment medical images datasets, acquired by $\mathrm{CT}$ or MRI, and create 3D vascular models, 


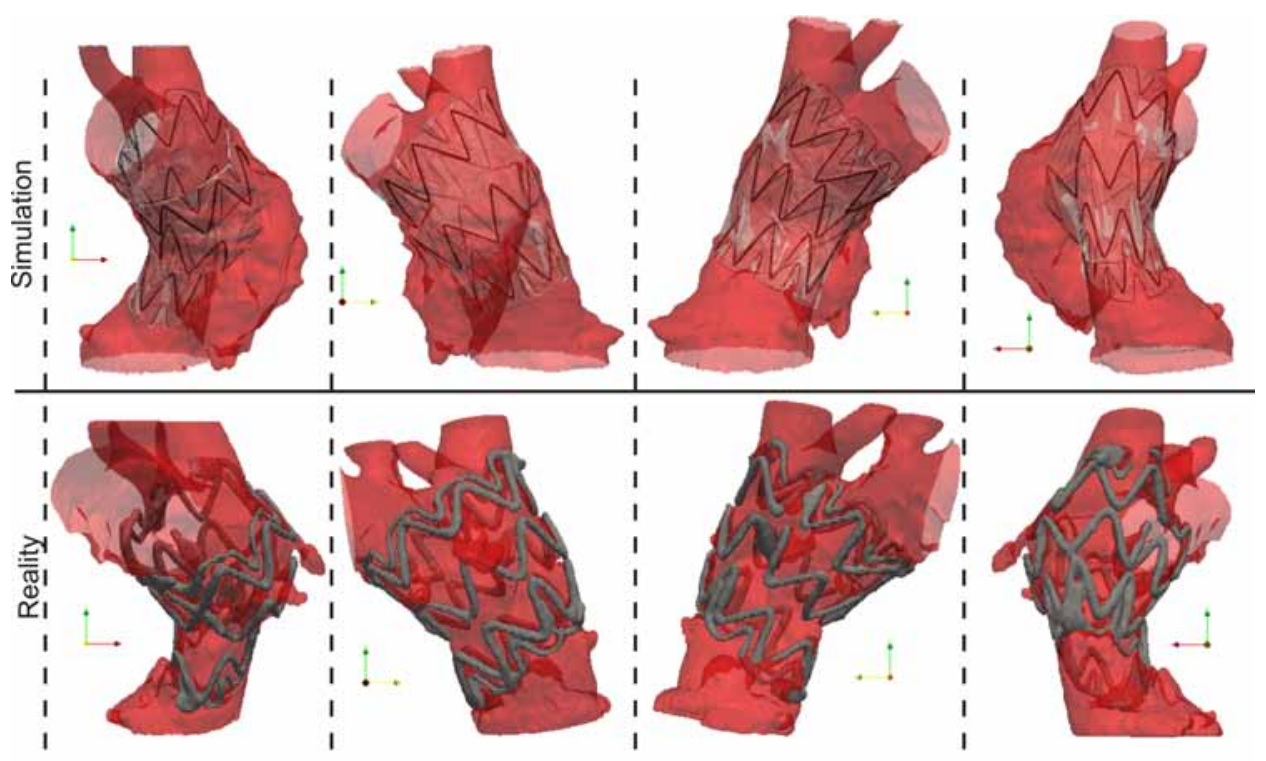

Figure 4. Finite element analysis, after TEVAR for treatment of ascending pseudoaneurysm. Adapted from Auricchio et al [26].

which can be further elaborated for the specific analysis purposes [19]. Besides the geometrical modeling, the preprocessing has the goal to extract preoperative physiological data to be used as analysis for boundary conditions. For instance, cine phase contrast MRI is adopted to compute inlet/outlet blood velocity profiles [18]. Doppler ultrasonography may obtain such information, although this technique cannot provide a velocity map because of arbitrarily oriented anatomic information.

\section{Solution}

The geometrical model and the boundary conditions should be consistent with the adopted solver technique, which computes the desired variables. Different numerical techniques are available: the selection of the computational methodology and of the related solver strictly depends on the type of problem under investigation. In cases involving the thoracic aorta, the following methodologies are currently under evaluation.

Structural finite element analysis (FEA). FEA is a numerical technique usually adopted in cardiovascular biomechanics to perform a virtual mechanical test for stent graft design or to evaluate the mechanics of implant performance with respect to the hosting biological environment (Fig. 4). Although structural FEA has been used extensively to investigate different aspects of coronary stenting or peripheral stenting [2025], very few studies address the structural behavior of aorta/graft interaction $[9,26,27]$. In particular, De Bock et al. [27] virtually deployed a bifurcated stent graft in an abdominal aortic aneurysm (AAA) model, using FEA, validating the simulation outcomes with respect to in vitro placement of the device in a silicone mock aneurysm, while in a more recent work, Auricchio et al. [26] compared the results of patient-specific FEA of ascending aorta endografting with postoperative images. Actually, only Figueroa et al. [9] have addressed the 3D pulsatile displacement forces acting on thoracic stent grafts using 3D computational techniques, combining computational fluid dynamics (CFD) and structural modeling. Nevertheless, the rapid extension of endovascular treatment of the thoracic aorta might increase FEA studies in this field, supporting the design of novel dedicated stent grafts or even supporting preoperative planning. The main limitation of these studies is that they rely on the assumptions regarding the arterial wall. The easiest choice is to assume the vessel wall as a rigid surface, which is reasonable for specific purposes and conditions; however, it is a strong simplification of a complex problem. In fact, modeling of the aortic wall, especially regarding pathological conditions like aortic aneurysm or dissection, is extremely challenging because the wall is inhomogeneous (healthy tissue plus diseased tissue plus thrombus). The standard approaches to calculate prestress are not so straightforward; neither is the assessment and assignment of material parameters related to phenomenological constitutive models [28- 


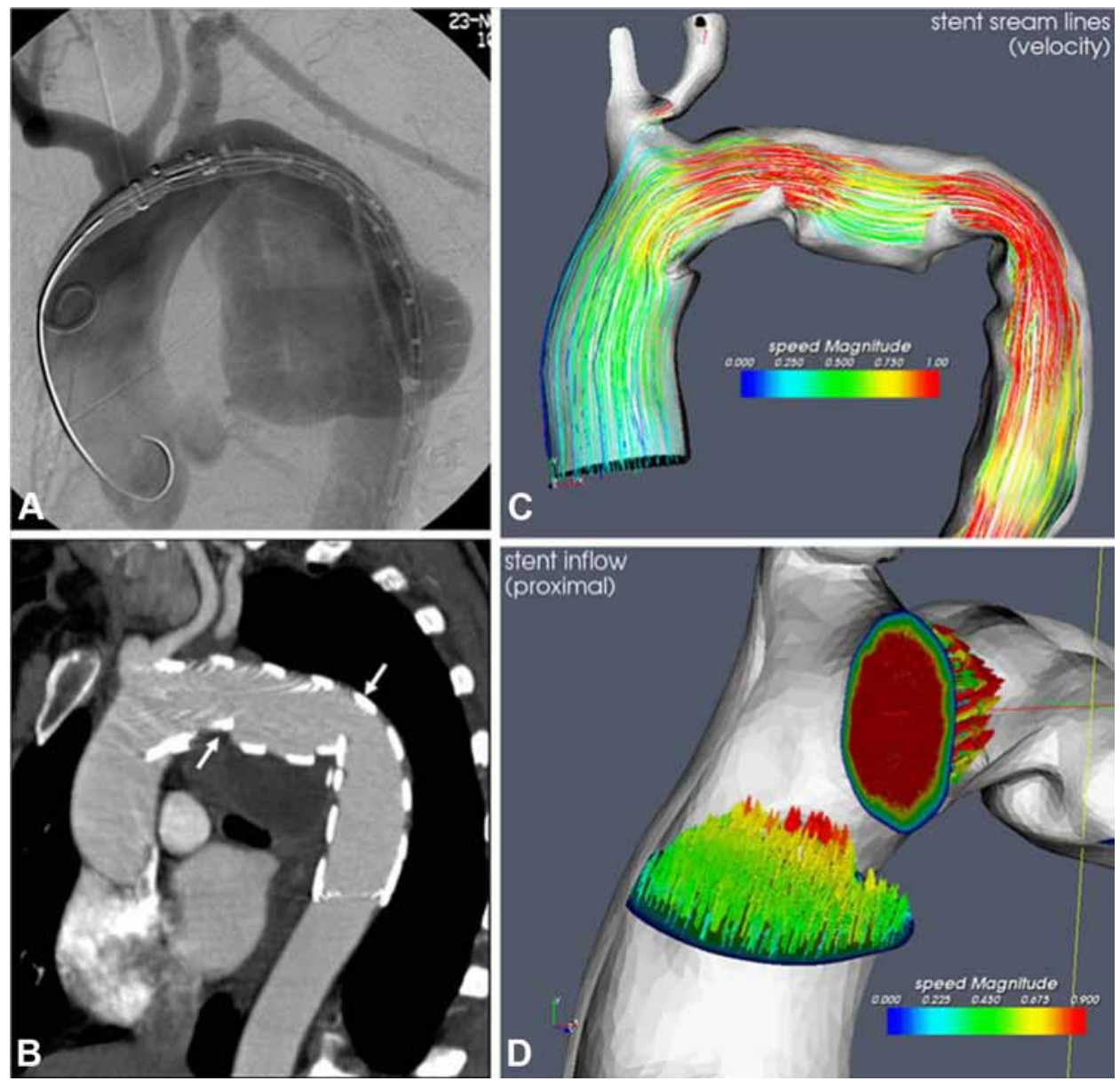

Figure 5. TEVAR for an aneurysm rupture at the isthmic region. A. Intraoperative angiography. B. Postoperative CTA with stent grafting of the aortic arch. C. Computational Fluid Dynamics. D. Vector-field analysis. Figure reproduced with permission of Springer, provided by Copyright Clearance Center. Adapted from Midulla et al [18].

30]. A new input toward the identification of patientspecific modeling of the aortic wall is the mechanical response, which can be derived from the analysis of 4D images, where the spatial information is coupled with the changes induced by the cardiac cycle, as proposed by Tierney et al. [31] in a recent study.

CFD. Computational Fluid Dynamics (CFD) is a numerical technique usually adopted in cardiovascular biomechanics to assess the hemodynamic conditions of a vessel segment (Fig. 5) [18]. An increasing number of studies regarding postoperative TEVAR hemodynamics are available in the literature [10,32-37]. Lam and colleagues $[32,34,35]$ in 2008 computed through CFD the drag force acting on a stent graft. Using the information obtained from CT imaging, they tailored an idealized model of the aorta and the implanted graft to a specific patient case, in order to investigate the impact of the stent graft apposition on the displacement force acting on the stent graft. Figueroa et al. [9], in 2009, assessed the displacement forces acting on thoracic stent grafts using CFD, proving that computational methods can enhance the understanding of the magnitude and orientation of the loads experienced in vivo by thoracic aortic stent grafts and therefore improve their design and performance (Fig. 6). In a similar manner, in 2011, Prasad et al. [36] evaluated through computer-based simulations the biomechanical and hemodynamic forces acting on the intermodular junctions of a multi-component thoracic stent graft, focusing on the development of type III endoleak due to disconnection of stent graft segments. Although the vessel wall displacement, calculated from the analysis of $4 \mathrm{D}$ images, can be imposed as a timedependent boundary condition, in CFD the vessel wall is 


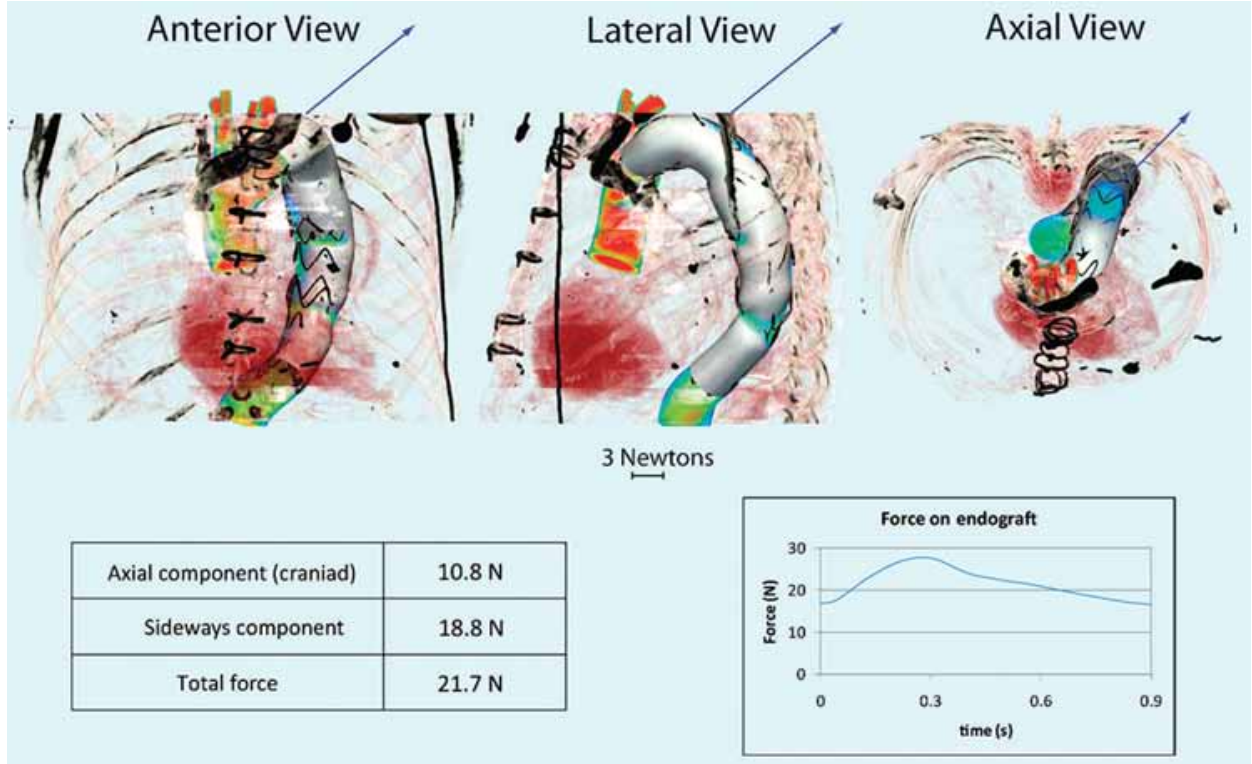

Figure 6. Mid-descending thoracic aortic endograft in anterior, lateral, and axial views showing the vector (arrow) of the displacement force (DF). Mean value of the DF vector, its sideways and axial components, and temporal variation over the cardiac cycle are given below. Note that the axial DF vector is in the cranial rather than the caudal direction. The DF magnitude changes over the cardiac cycle, varying from $16.7 \mathrm{~N}$ in diastole and $27.8 \mathrm{~N}$ at peak systole. Figure reproduced with permission of the Journal of Endovascular Therapy (৫ 2009), provided by Copyright Clearance Center. International Society of Endovascular Specialists. Adapted from Figueroa et al [9].

basically assumed to be rigid, neglecting the mutual interaction between the vascular wall and the pulsatile action of the blood [38].

It is worth noting that, as already reported, the simulation outcomes are strictly related to the assumed boundary conditions, with flow area at the inlet and pressure at the outlets $[39,40]$.

Most cardiovascular phenomena and mechanisms rely on a close and mutual interaction between the blood and the vessel; for instance, the dissection flap floating due to the action of blood flow. Consequently, many biomechanical analyses regarding the cardiovascular biomechanics are based on fluidstructure interaction (FSI), which is the generic name referring to a family of numerical techniques aimed at solving the coupled problem given by interaction between a deformable solid body (eg, the vessel wall) and a fluid (eg, blood). FSI has been widely used to investigate the aortic biomechanics; however, due to the involved modeling complexity, no studies have previously addressed the thoracic stent grafting $[41,42]$. Some authors idealized the mechanical response of the stent graft deployed in an abdominal aneurysm, reconstructed from CTA, within FSI $[43,44]$.

\section{Postprocessing: From Numerical Outputs to Clinical Relevant Information}

Numerical simulations produce a huge amount of data, which should be properly elaborated to provide few significant indices, resuming clinical relevant information. Although stress distribution along the vessel wall is usually obtained from FEA or FSI analyses, many sophisticated hemodynamic indices are currently available with different purposes: for example, the calculation of the helicity coefficient of the aortic flow, computed from 4D MRI, suggests that aortic helical flow might be caused by natural optimization of fluid transport processes in the cardiovascular system, aimed at obtaining efficient perfusion $[45,46]$. Morbiducci et al. [46] studied the hemodynamics in the aorta in 5 healthy volunteers. They used 4D PCMRI in combination with computational analysis of advanced fluid dynamics. These algorithms enabled construction of a 4D representation to visualize the aortic helical flow, which is a result of the alignment of velocity and vorticity (Fig. 7) [46]. The main findings in the literature of the three different numerical techniques (FEA, CFD, and FSI) are demonstrated in Table 1. 


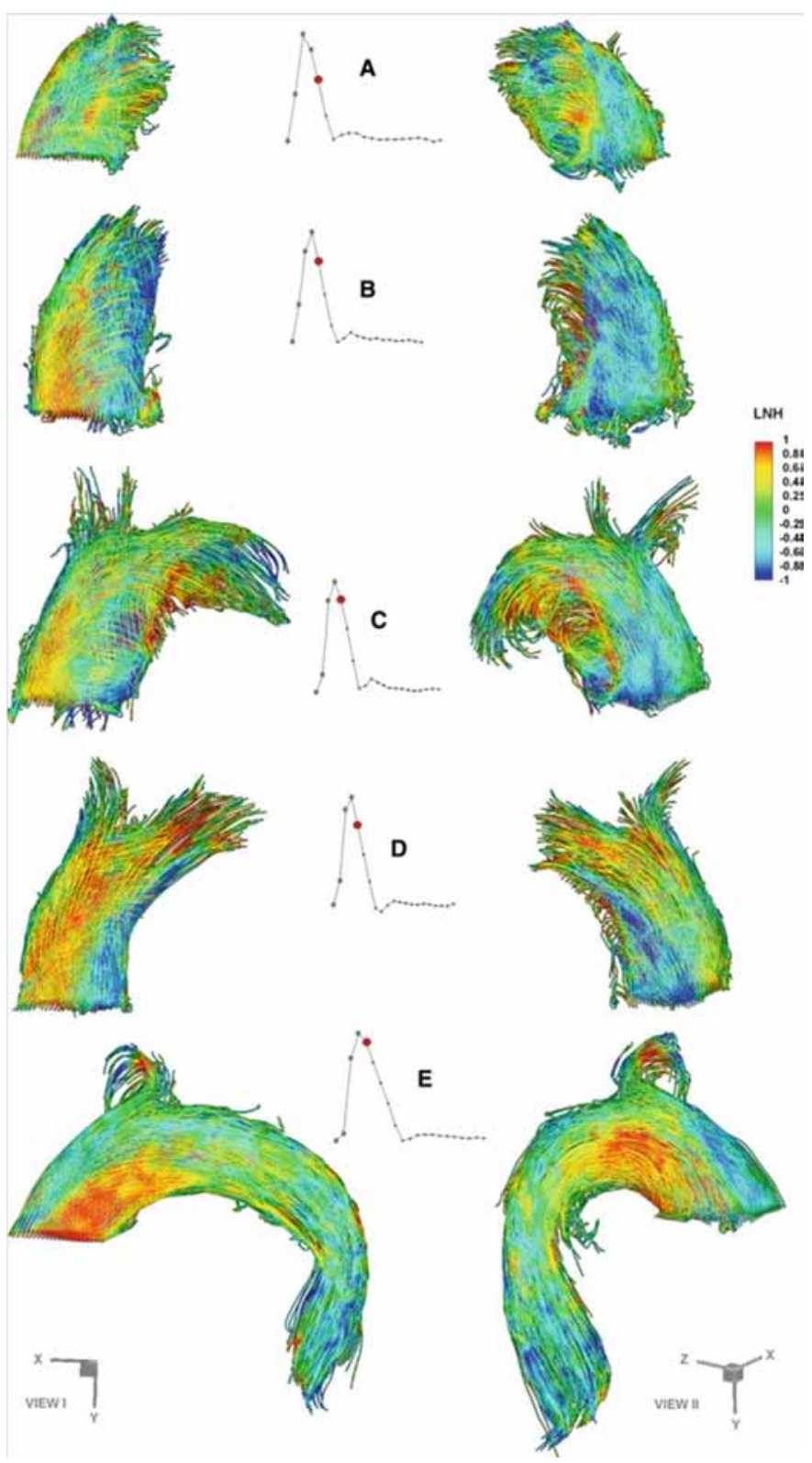

Figure 7. Evolution of the helical flow in the 5 different aortas (two different views). The flow in $A$ and $E$ has a helical structure and the flow in $B, C$, and $D$ has a bihelical structure. Figure reproduced with permission of Springer, provided by Copyright Clearance Center. Adapted from Morbiducci et al [46].

\section{Computational Analysis Integrated in Imaging Modalities}

Different computational analysis models have been used for hemodynamic aortic analysis; they have been used especially to create simulations of the blood flow in the abdominal aorta before and after stent graft placement $[47,48]$. Frydrychowicz et al. [49] stated the poten- tial of 4D PC-MRI, in which computational analysis can be easily integrated using the steps of simulation of preprocessing, solution, and postprocessing. 4D PC-MRI includes velocity in 3 directions encoding in $3 \mathrm{D}$ imaging over time, with the potential to extensively analyze human physiologic hemodynamics in vivo. 4D PC-MRI is used especially in organ hemodynamics where complex flows are present, as in cardiovascular disease and neurovascular imaging. Because of the lack of ionizing radiation, MRI can be widely used, although at present it is only available in some specialized centers. Due to the relatively short acquisition time of this new imaging modality, it can be adopted in the clinical practice to evaluate most patients and disease-related questions [49]. 4D PC-MRI offers a large amount of information, enabling the measurement of different parameters, such as pulse wave velocity (PWV), the speed at which the pulse wave travels through the vessel or particular system, and estimates of wall shear stress (WSS), which is the frictional force created by the circulating blood (Fig. 8) [49]. Another recent study reported the combined information of CFD and MRI for functional analysis of the thoracic aorta after stent graft implantation, showing the usefulness of the technique by visualization of patient-specific aortic hemodynamics with the blood flow pattern and local pathologic changes [18].

Through computational modeling, complications after stent graft placement might be detected, as has already been shown for endovascular abdominal aneurysm repair (EVAR) $[47,48]$.

\section{Future Perspectives and Conclusions}

Currently, stent graft sizing is performed using static $\mathrm{CT}$ or MRI imaging. In order to improve endovascular treatment decision-making and outcome, three-dimensional volumetric analysis during the cardiac cycle is needed. Computational modeling is still in the experimental phase, but it gradually provides insight into the causative mechanisms of stent graft complications and the forces experienced in vivo by thoracic aortic stent grafts. A better understanding of this process may lead to change in stent graft design, with the aim to increase performance. In the future, 3D computational models, simulating the in vivo environment, may be used in daily practice to test endovascular stent graft behavior, both preoperatively, and during follow-up [50].

Computational analysis for thoracic aortic disease is a promising method to study the hemodynamics, with dif- 
Table 1. Main findings and references for the three numerical techniques: FEA, CFD, and FSI

\begin{tabular}{|c|c|c|}
\hline & Main findings & References \\
\hline \multirow[t]{3}{*}{ FEA } & $\begin{array}{l}\text { Validation of bifurcated stent graft deployment in AAA } \\
\text { with an in vitro model }\end{array}$ & De Bock et al. 2012 [27] \\
\hline & $\begin{array}{l}\text { Comparison of patient-specific FEA with post-operative } \\
\text { images after TEVAR }\end{array}$ & Auricchio et al. 2013 [26] \\
\hline & $\begin{array}{l}\text { Assessment of 3D pulsatile displacement forces acting on } \\
\text { thoracic stent grafts }\end{array}$ & Figueroa et al. 2009 [9] \\
\hline \multirow[t]{4}{*}{ CFD } & $\begin{array}{l}\text { Assessment of the hemodynamic conditions of a vessel } \\
\text { segment }\end{array}$ & Midulla et al. 2012 [18] \\
\hline & Assessment of the hemodynamics of the aorta after TEVAR & $\begin{array}{l}\text { Figueroa et al. } 2011 \text { [10]; Fung et al. } 2008 \text { [32]; Gallo et al. } \\
2012 \text { [33]; Lam et al. } 2007 \text { [34]; Lam et al. } 2008 \text { [35]; } \\
\text { Prasad et al. } 2011 \text { [36]; Tse et al. } 2011 \text { [37] }\end{array}$ \\
\hline & $\begin{array}{l}\text { Estimation of the displacement forces acting on thoracic } \\
\text { stent graft (measured using CTA) }\end{array}$ & $\begin{array}{l}\text { Figueroa et al. } 2009 \text { [9]; Fung et al. } 2008 \text { [32]; Lam et al. } \\
2007 \text { [34]; Lam et al. } 2008 \text { [35]; Prasad et al. } 2011 \text { [36] }\end{array}$ \\
\hline & 4D representation to visualize the aortic helical flow & Morbiducci et al. 2011 [46] \\
\hline \multirow[t]{2}{*}{ FSI } & Aortic blood flow simulations & Bazilevs et al. 2006 [41]; Crosetto et al. 2011 [42] \\
\hline & $\begin{array}{l}\text { Assessment of the mechanical response of the stent graft } \\
\text { in AAA reconstructed from CTA }\end{array}$ & Li et al. 2006 [43]; Molony et al. 2010 [44] \\
\hline
\end{tabular}

AAA indicates abdominal aortic aneurysm; CFD, computational fluid dynamics; CTA, computed tomography angiography; FEA, finite element analysis; FSI, fluid structure interaction; TEVAR, thoracic endovascular aortic repair.
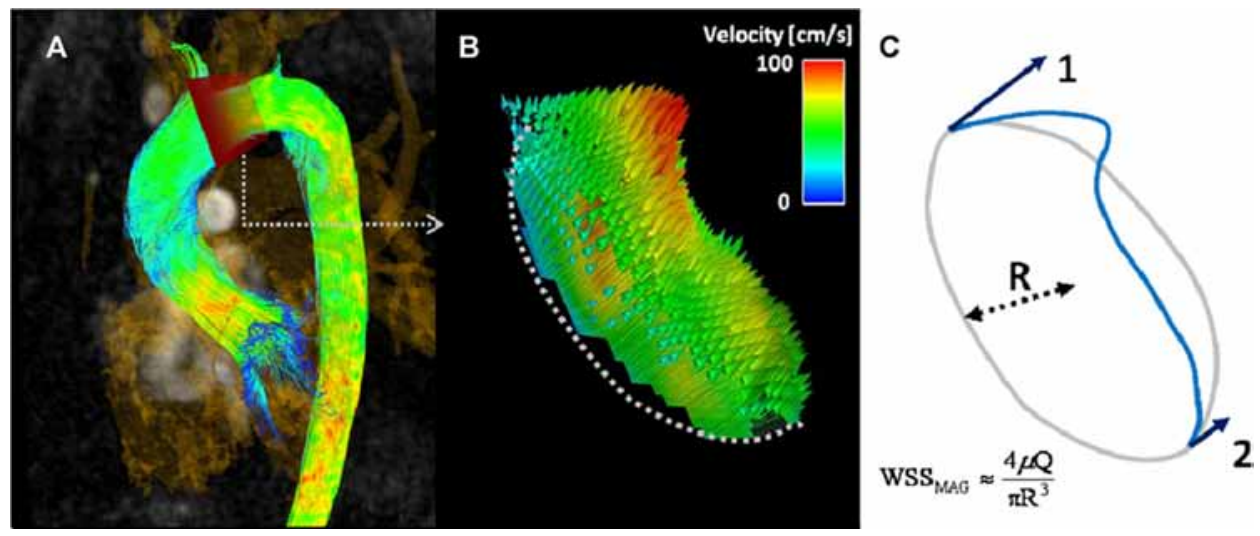

Figure 8. Wall shear stress (WSS) is calculated from the 4D PC-MRI data. Figure reproduced with permission of Elsevier, provided by Copyright Clearance Center. Adapted from Frydrychowicz et al [49].

ferent possible clinical applications. Preoperative measurements regarding the inflow rate and flow split along the aortic branches can be retrieved from echocardiographic images or echo-Doppler measurements. Such measurements can be used as boundary conditions for the fluid dynamics analysis. Similarly, the postoperative and follow-up flow rate and flow split can be used for validation purposes. Computational analysis can complement MRI or $\mathrm{CT}$ imaging, providing hemodynamic parameters and mechanical forces that are not available in routine clinical imaging. In addition, computational analysis might simulate virtual treatment preoperatively in order to predict the outcome of TEVAR. The computational models need to be validated and more refined before they can be fully implemented in clinical practice, but the outlook is promising.

\section{Acknowledgments}

Michele Conti, PhD, acknowledges The ERC Starting Grant Project ISOBIO: Isogeometric Methods for Biomechanics (No. 259229).

\section{Comment on this Article or Ask a Question}




\section{References}

1. Grabenwöger M, Alfonso F, Bachet J, Bonser $\mathrm{R}$, Czerny M, Eggebrecht $\mathrm{H}$, et al. Thoracic Endovascular Aortic Repair (TEVAR) for the treatment of aortic diseases: a position statement from the European Association for Cardio-Thoracic Surgery (EACTS) and the European Society of Cardiology (ESC), in collaboration with the European Association of Percutaneous Cardiovascular Interventions (EAPCI). Eur Heart J. 2012;33:15581563. 10.1093/eurheartj/ehs074

2. Hiratzka LF, Bakris GL, Beckman JA, Bersin RM, Carr VF, Casey DE, Jr, et al. 2010 ACCF/ AHA/AATS/ACR/ASA/SCA/SCAI/SIR/STS/SVM Guidelines for the diagnosis and management of patients with thoracic aortic disease. A Report of the American College of Cardiology Foundation/American Heart Association Task Force on Practice Guidelines, American Association for Thoracic Surgery, American College of Radiology, American Stroke. Association, Society of Cardiovascular Anesthesiologists, Society for Cardiovascular Angiography and Interventions, Society of Interventional Radiology, Society of Thoracic Surgeons, and Society for Vascular Medicine. J Am Coll Cardiol. 2010;55:e27e129. 10.1016/j.jacc.2010.02.015

3. Muhs BE, Vincken KL, van Prehn J, Stone MK, Bartels LW, Prokop M, et al. Dynamic cine-CT angiography for the evaluation of the thoracic aorta; insight in dynamic changes with implications for thoracic endograft treatment. Eur J Vasc Endovasc Surg. 2006;32: 532-536. 10.1016/j.ejvs.2006.05.009

4. van Keulen JW, van Prehn J, Prokop M, Moll $\mathrm{FL}$, van Herwaarden JA. Dynamics of the aorta before and after endovascular aneurysm repair: a systematic review. Eur J Vasc Endovasc Surg. 2009;38:586-596. 10.1016/j. ejvs.2009.06.018

5. van Prehn J, Vincken KL, Muhs BE, Barwegen GK, Bartels LW, Prokop M, et al. Toward endografting of the ascending aorta: insight into dynamics using dynamic cine-CTA. J Endovasc Ther. 2007;14:551-560. 10.1583/ 1545-1550(2007)14[551:TEOTAA]2.0.CO;2

6. van Prehn J, Vincken $\mathrm{KL}$, Sprinkhuizen $\mathrm{SM}$ Viergever MA, van Keulen JW, van Herwaarden JA, et al. Aortic pulsatile distention in young healthy volunteers is asymmetric: analysis with ECG-gated MRI. Eur J Vasc Endovasc Surg. 2009;37:168-174.10.1016/j.ejvs. 2008.11.007

7. van Prehn J, Bartels LW, Mestres G, Vincken $\mathrm{KL}$, Prokop M, Verhagen $\mathrm{HJ}$, et al. Dynamic aortic changes in patients with thoracic aortic aneurysms evaluated with electrocardiography-triggered computed tomographic angiography before and after thoracic endovascular aneurysm repair: preliminary re- sults. Ann Vasc Surg. 2009;23:291-297. 10. 1016/j.avsg.2008.08.007

8. Lin KK, Kratzberg JA, Raghavan ML. Role of aortic stent graft oversizing and barb characteristics on folding. J Vasc Surg. 2012;55: 1401-1409. 10.1016/j.jvs.2011.10.080

9. Figueroa CA, Taylor CA, Chiou AJ, Yeh V, Zarins CK. Magnitude and direction of pulsatile displacement forces acting on thoracic aortic endografts. J Endovasc Ther. 2009;16: 350-358. 10.1583/09-2738.1

10. Figueroa CA, Zarins CK. Computational analysis of displacement forces acting on endografts used to treat aortic aneurysms. In Biomechanics and Mechanobiology of Aneurysms (McGloughlin T, ed). XIV. Springer. 2011;221-246.

11. Hsiao EM, Rybicki FJ, Steigner M. CT coronary angiography: 256-slice and 320-detector row scanners. Curr Cardiol Rep. 2010;12: 68-75. 10.1007/s11886-009-0075-Z

12. Sabarudin A, Sun Z, Yusof AK. Coronary CT angiography with single-source and dualsource $\mathrm{CT}$ : comparison of image quality and radiation dose between prospective ECGtriggered and retrospective ECG-gated protocols. Int J Cardiol. 2012 Oct. 22. 10.1016/j. ijcard.2012.09.217

13. Willemink MJ, Habets J, de Jong PA, Schilham AM, Mali WP, Leiner $T$, et al. Iterative reconstruction improves evaluation of native aortic and mitral valves by retrospectively ECG-gated thoracoabdominal CTA. Eur Radiol.2013;4:968-974.10.1007/s00330-0122673-1

14. Ko JP, Brandman S, Stember J, Naidich DP. Dual-energy computed tomography: concepts, performance, and thoracic applications. J Thorac Imaging. 2012;27:7-22. 10. 1097/RTI.0b013e31823fe0e9

15. Frydrychowicz A, Berger A, Munoz Del Rio A, Russe MF, Bock J, Harloff $A$, et al. Interdependencies of aortic arch secondary flow patterns, geometry, and age analysed by 4-dimensional phase contrast magnetic resonance imaging at 3 Tesla. Eur Radiol. 2012; 22:1122-1130. 10.1007/s00330-011-2353-6

16. Markl M, Frydrychowicz A, Kozerke S, Hope M, Wieben O. 4D flow MRI. J Magn Reson Imaging. 2012;36:1015-1036. 10.1002/jmri. 23632

17. Taylor CA, Figueroa CA. Patient-specific modeling of cardiovascular mechanics. Annu Rev Biomed Eng. 2009;11:109-134. 10. 1146/annurev.bioeng.10.061807.160521

18. Midulla M, Moreno R, Baali A, Chau M, Negre-Salvayre A, Nicoud F, et al. Haemodynamic imaging of thoracic stent-grafts by computational fluid dynamics (CFD): presentation of a patient-specific method combining magnetic resonance imaging and nu- merical simulations. Eur Radiol. 2012;22: 2094-2102. 10.1007/s00330-012-2465-7

19. De Santis G, De Beule M, Van Canneyt K, Verdonck $P$, Verhegghe $B$. Full-hexahedral structured meshing for image-based computational vascular modeling. Med Eng Phys. 2011;10:1318-1325. 10.1016/j. medengphy.2011.06.007

20. Auricchio F, Di Loreto M, Sacco E. Finite element analysis of a stenotic artery revascularization through stent insertion. Computer Methods in Biomechanics and Biomedical Engineering. 2001;4:249-263. 10. 1080/10255840108908007

21. Auricchio F, Conti M, De Beule M, De Santis G, Verhegghe B. Carotid artery stenting simulation: from patient-specific images to finite element analysis. Med Eng Phys. 2011; 33:281-289. 10.1016/j.medengphy.2010.10. 011

22. Conti M, Van Loo D, Auricchio F, De Beule M, De Santis G, Verhegghe $B$, et al. Impact of carotid stent cell design on vessel scaffolding: a case study comparing experimental investigation and numerical simulations. J Endovasc Ther. 2011;18:397-406.10.1583/103338.1

23. Gijsen FJ, Migliavacca F, Schievano S, Socci L, Petrini L, Thury A, et al. Simulation of stent deployment in a realistic human coronary artery. Biomed Eng Online. 2008;7:23. 10 1186/1475-925X-7-23

24. Lally C, Dolan F, Prendergast PJ. Cardiovascular stent design and vessel stresses: a finite element analysis. J Biomech. 2005;38: 1574-1581. 10.1016/j.jbiomech.2004.07.022 25. Mortier P, Holzapfel GA, De Beule M, Van Loo D, Taeymans $Y$, Segers $P$, et al. A novel simulation strategy for stent insertion and deployment in curved coronary bifurcations: comparison of three drug-eluting stents. Ann Biomed Eng. 2010;38:88-99. 10.1007/ s10439-009-9836-5

26. Auricchio F, Conti M, Marconi S, Reali A, Tolenaar JL, Trimarchi S. Patient-specific aortic endografting simulation: from diagnosis to prediction. Comput Biol Med. 2013;43: 386-394. 10.1016/j.compbiomed.2013.01. 006

27. De Bock $S$, lannaccone $F$, De Santis G, De Beule M, Van Loo D, Devos D, et al. Virtual evaluation of stent graft deployment: a validated modeling and simulation study. J Mech Behav Biomed Mater. 2012;13:129_ 139. 10.1016/j.jmbbm.2012.04.021

28. Alastruè V, Garcia A, Pena E, Rodriguez J, Martinez M, Doblare M. Numerical framework for patient-specific computationa modelling of vascular tissue. Int J Numerical Methods Biomed Eng. 2010;26:35-51. 10. 1002/cnm.1234 
29. Gee M, Förster C, Wall W. A computational strategy for prestressing patient-specific biomechanical problems under finite deformations. Int J Numerical Methods Biomed Eng. 2010;26:52-72. 10.1002/cnm.1236

30. Holzapfel GA, Gasser T, Ogden R. A new constitutive framework for arterial wall mechanics and a comparative study of material models. J Elasticity. 2000;61:1-48. 10.1023/A: 1010835316564

31. Tierney ÁP, Callanan A, McGloughlin TM. Use of regional mechanical properties of abdominal aortic aneurysms to advance finite element modeling of rupture risk. J Endovasc Ther. 2012;1:100-114. 10.1583/11-3456.1

32. Fung GS, Lam SK, Cheng SW, Chow KW. On stent-graft models in thoracic aortic endovascular repair: a computational investigation of the hemodynamic factors. Comput Biol Med. 2008;38:484-489. 10.1016/j. compbiomed.2008.01.012

33. Gallo D, De Santis G, Negri F, Tresoldi D, Ponzini $R$, Massai $D$, et al. On the use of in vivo measured flow rates as boundary conditions for image-based hemodynamic models of the human aorta: implications for indicators of abnormal flow. Ann Biomed Eng. 2012;40:729-741. 10.1007/ s10439-011-0431-1

34. Lam SK, Fung GS, Cheng SW, Chow KW. A computational investigation on the effect of biomechanical factors related to stent-graft models in the thoracic aorta. Conf Proc IEEE Eng Med Biol Soc. 2007;943-946. 10.1007/ s11517-008-0361-8

35. Lam SK, Fung GS, Cheng SW, Chow KW. A computational study on the biomechanical factors related to stent-graft models in the thoracic aorta. Med Biol Eng Comput. 2008; 46:1129-1138. 10.1007/s11517-008-0361-8

36. Prasad A, To LK, Gorrepati ML, Zarins CK, Figueroa CA. Computational analysis of stresses acting on intermodular junctions in thoracic aortic endografts. J Endovasc Ther. 2011;18:559-568. 10.1583/11-3472.1

37. Tse KM, Chiu P, Lee HP, Ho P. Investigation of hemodynamics in the development of dissecting aneurysm within patient-specific dissecting aneurismal aortas using computational fluid dynamics (CFD) simulations. J Biomech. 2011;44:827-836. 10. 1016/j.jbiomech.2010.12.014

38. Stalder AF, Liu Z, Hennig J, Korvink JG, Li KC, Markl M. Patient specific hemodynamics: combined 4D flow-sensitive MRI and CFD. Computational Biomechanics for Medicine. XII. Springer, 2010. p. 27-38. 10.1007/978-14419-9619-0_4

39. Balossino R, Pennati G, Migliavacca F, Formaggia L, Veneziani A, Tuveri M, et al. Computational models to predict stenosis growth in carotid arteries: which is the role of boundary conditions? Comput Methods Biomech Biomed Eng.2009;12:113-123.10.1080/ 10255840903080802

40. Vignon-Clementel IE, Figueroa CA, Jansen $\mathrm{KE}$, Taylor CA. Outflow boundary conditions for three-dimensional finite element modeling of blood flow and pressure in arteries. Comput Methods Appl Mech Engrg. 2006; 195:3776-3796. 10.1016/j.cma.2005.04.014

41. Bazilevs Y, Calo VM, Zhang Y, Hughes TJR. Isogeometric fluid structure interaction analysis with applications to arterial blood flow. Comput Mech. 2006;38:310-322. 10.1007/ s00466-006-0084-3

42. Crosetto P, Reymond P, Deparis S, Kontaxakis V, Stergiopulos N, Quarteroni A. Fluidstructure interaction simulation of aortic blood flow. Comp Fluids. 2011;43:46-57. 10. 1016/j.compfluid.2010.11.032

43. Li Z, Kleinstreuer C. Computational analysis of type II endoleaks in a stented abdominal aortic aneurysm model. J Biomech. 2006;39: 2573-2582. 10.1016/j.jbiomech.2005.09.002

44. Molony DS, Kavanagh EG, Madhavan P, Walsh MT, McGloughlin TM. A computational study of the magnitude and direction of migration forces in patient-specific abdominal aortic aneurysm stent-grafts. Eur J Vasc Endovasc Surg. 2010;40:332-339. 10. 1016/j.ejvs.2010.06.001

45. Borghi A, Wood NB, Mohiaddin RH, Xu XY. Fluid-solid interaction simulation of flow and stress pattern in thoracoabdominal aneurysms: a patient-specific study. J Fluids Structures 2008;24:270-280. 10.1016/j. jfluidstructs.2007.08.005

46. Morbiducci U, Ponzini R, Rizzo G, Cadioli M, Esposito A, Montevecchi FM, et al. Mechanistic insight into the physiological relevance of helical blood flow in the human aorta: an in vivo study. Biomech Model Mechanobiol. 2011;10:339-355. 10.1007/ s10237-010-0238-2

47. Frauenfelder T, Lotfey M, Boehm T, Wildermuth S. Computational fluid dynamics: hemodynamic changes in abdominal aortic aneurysm after stent-graft implantation. Cardiovasc Intervent Radiol. 2006;29:613-623. 10.1007/s00270-005-0227-5

48. Howell BA, Kim T, Cheer A, Dwyer H, Saloner D, Chuter TA. Computational fluid dynamics within bifurcated abdominal aortic stentgrafts. J Endovasc Ther. 2007;14:138-143. 10.1583/1545-1550(2007)14[138:CFDWBA]2. $0 . \mathrm{CO} ; 2$

49. Frydrychowicz A, Francois CJ, Turski PA. Four-dimensional phase contrast magnetic resonance angiography: potential clinical applications. Eur J Radiol. 2011;80:24-35. 10. 1016/j.ejrad.2011.01.094

50. Zarins CK, Taylor CA. Endovascular device design in the future: transformation from trial and error to computational design. J Endovasc Ther. 2009;16(Suppl 1):I12-121. 10. 1583/08-2640.1

Cite this article as: van Bogerijen GHW, Tolenaar JL, Conti M, Auricchio F, Secchi F, Sardanelli F, Moll FL, van Herwaarden JA, Rampoldi V, Trimarchi S. Contemporary Role of Computational Analysis in Endovascular Treatment for Thoracic Aortic Disease. Aorta 2013;1(3):171-181. DOI: http://dx.doi. org/10.12945/j.aorta.2013.13-003 